\title{
Consumo de alcohol entre estudiantes mexicanos de medicina
}

\author{
Angel Puig-Nolasco ${ }^{1}$ \\ Leticia Cortaza-Ramirez ${ }^{2}$ \\ Sandra Cristina Pillon ${ }^{3}$
}

\begin{abstract}
Este estudio tiene por objetivo identificar el patrón de consumo de alcohol entre los estudiantes mexicanos de medicina. Se trata de un estudio transversal realizado en la Universidad Veracruzana, en México, con 263 estudiantes (84,4\%). El cuestionario contiene la Prueba de Identificación de Trastornos por el Uso del Alcohol y el Cuestionario de Estudiantes 2006. Los sujetos eran $60 \%$ mujeres, jóvenes. El uso en la vida fue de $71,9 \%$ y la edad de inicio 12,5 años. La frecuencia del consumo fue baja pero en alta cantidad, consumen en niveles de emborrachamiento, principalmente los hombres. El $46 \%$ consume bebidas en niveles problemáticos. Los estudiantes tienen la percepción de que los padres y maestros ven muy mal que ellos consuman bebidas alcohólicas. Este estudio tiene contribuciones para el planteamiento de programas preventivos en relación al consumo de bebidas alcohólicas para ser aplicados en el ámbito universitario.
\end{abstract}

Descriptores: Consumo de Bebidas Alcohólicas; Estudiantes de Medicina; Percepción.

\footnotetext{
${ }^{1}$ Doctor en Neuroetologia, Profesor de Tiempo Completo, Facultad de Medicina, Universidad Veracruzana, Campus Minatitlán, Veracruz, México. E-mail: apuign@hotmail.com.

${ }^{2}$ Doctor en Enfermería, Profesor de Tiempo Completo, Facultad de Enfermería, Universidad Veracruzana, Campus Veracruz, México. E-mail: leticortaza@hotmail.com.

${ }^{3}$ Enfermera, Doctora en Enfermería. Profesor Asociado, Escola de Enfermagem de Ribeirão Preto, Universidade de São Paulo, Centro Colaborador de la OMS para el Desarrollo de la Investigación en Enfermería, SP, Brasil. E-mail: pillon@eerp.usp.br.
}

Correspondencia:

Sandra Cristina Pillon

Universidade de São Paulo. Escola de Enfermagem de Ribeirão Preto

Departamento de Enfermagem Psiquiátrica e Ciências Humanas

Av. dos Bandeirantes, 3900

Bairro: Monte Alegre

CEP: 14040-902 Ribeirão Preto, SP, Brasil

E-mail: pillon@eerp.usp.br 


\section{O uso de álcool entre estudantes de medicina mexicanos}

O estudo teve por objetivo identificar o padrão de consumo de álcool entre estudantes de medicina mexicanos. Trata-se de estudo transversal, realizado na Universidade Veracruzana, México, com 263 estudantes (84.4\%). Foram usados o questionário do teste de identificação do uso de álcool e o questionário de estudantes 2006. Os sujeitos eram mulheres jovens. O uso na vida foi de $71,9 \%$ e a idade de início 12,5 anos. A frequência de consumo foi baixa, mas em alta quantidade, com padrão de embriaguez, principalmente entre os homens. Dos estudantes, $46 \%$ consomem bebidas alcoólicas em níveis problemáticos. Os estudantes percebem que os pais e professores avaliam muito mal o fato de eles consomirem bebidas alcoólicas. Este estudo traz contribuições para o planejamento de programas preventivos em relação ao consumo de bebidas alcoólicas, no âmbito universitário.

Descritores: Consumo de Bebidas Alcoólicas; Estudantes de Medicina; Percepção.

\section{Alcohol Use Among Mexican Medical Students}

The study aims to identify patterns of alcohol consumption in Mexican medical students. This is a cross-sectional study, conducted at the University of Veracruz, Mexico. The sample comprised 263 students (84.4\%). The Alcohol Use Disorders Identification Test and the Student Questionnaire 2006 were used. Students were characterized by youth and women. Seventy-two percent used alcohol in the lifetime, with 12.5 years as the onset age. The frequency of consumption was low but the quantity high, with a pattern of drunkenness, mainly among men. Forty-six percent consumed beverage at a problematic level. Students perceive that parents and teachers badly assess alcoholic beverage consumption. This study contributes to the planning of preventive programs for alcohol consumption, to be applied in the university context.

Descriptors: Alcohol Drinking; Students, Medical; Perception.

\section{Introducción}

En México el abuso en el consumo de alcohol constituye un creciente y preocupante problema de Salud Publica; el alcohol es una sustancia potencialmente adictiva utilizada con mayor frecuencia por los adultos jóvenes, específicamente entre aquellos en edad de recibir una educación universitaria o superior. El consumo de alcohol en estudiantes ha generado interés desde una óptica de Salud Pública, por los problemas relacionados con su consumo(1-3).

En este país desde 1988 el consumo de alcohol por la población general viene siendo monitoreada por las Encuestas Nacionales de Adicciones, así los datos muestran que de 1988 a 2008 la prevalencia de uso en la vida en mayores de 18 años varió de 40,4\% a $70 \%$, así como la edad de inicio de consumo se viene

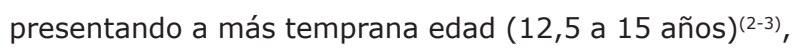

indicando que ese consumo empieza antes de los 18 años, un periodo considerado prohibido legalmente para venta y consumo.

En la referida encuesta, el consumo de bebidas alcohólicas en México fue mencionado como un importante problema de salud pública, particularmente entre los jóvenes que de forma precoz inician su consumo. Además, los estudiantes universitarios forman un grupo que llama la atención a los educadores y profesionales de salud. Al respecto la literatura señala que el consumo de bebidas alcohólicas entre estos jóvenes ha sido muy alto(4).

En este sentido, estudios anteriores evidenciaron que los estudiantes del área de la salud constituyen un grupo de población que merece una atención especial en relación al uso del alcohol y de otras sustancias, 
pues representan a los profesionales que, en el futuro, trabajaran en cuestiones de salud en la comunidad(5).

En países de América latina las evidencias muestran índices de prevalencia preocupantes sobre el uso en la vida en estudiantes de diferentes facultades de medicina $50 \%$ a $86,9 \%$ en Brasil( ${ }^{(6-8)}$ y $89,3 \%$ a $98,1 \%$ en Colombia( ${ }^{(9-10)}$ y otros países como Irlanda $71,2 \%{ }^{(11)}$.

Estudios anteriores en estudiantes colombianos del área de salud (Medicina, Odontología y Enfermería) observaron: consumo de alcohol en niveles perjudiciales en $34,5 \%$. Dentro de los problemas relacionados al consumo de alcohol, los problemas físicos (cefaleas, gastritis, vómitos, fotofobia) fueron los más prevalentes, así como la relaciones sexuales no planificadas o sin protección bajo los efectos del alcohol; problemas familiares como causa del consumo de alcohol; enfrentamientos físicos o problemas con la autoridad o la policía y con el grupo social; y problemas académicos en ambos sexos ${ }^{(10)}$.

Al evaluar estudiantes de medicina por medio de la Prueba de Identificación de Trastornos por el uso del alcohol (AUDIT) se identificó que el 44,2\% tuvo algún tipo de uso problemático de alcohol (puntuación $\geq 8$ en el AUDIT), siendo 35,7\% mujeres y 53,1\% hombres. En relación a la frecuencia de consumo de alcohol 45,5\% de los universitarios refirieron que consumían de dos a cuatro veces por mes, $17 \%$ bebían de dos a tres veces por semana, $29,7 \%$ en cantidad de tres a cuatro dosis y 33,9 más de 5 dosis ${ }^{(12)}$ datos considerados preocupantes una vez que la mayoría eran mujeres.

En cuanto a los motivos por el cual los estudiantes consumen drogas y bebidas alcohólicas en altas cantidades son: tener tiempo libre y sentimientos como aburrimiento, tener amigos o haber cometido actos antisociales. Respecto a la familia, manifiestan cumplir menos con las normas parentales y muestran menor interés en hacerlo. Así, un porcentaje más elevado informó que sus padres han: peleado con frecuencia, pedido ayuda por esa razón o intentado separarse ${ }^{(13)}$.

Los sentimientos de estudiantes universitarios al conocer que un familiar suyo consume algún tipo de droga, fueron que: 21 (50\%) sienten rechazo/ indignación y $17(40,5 \%)$ conformismo. Considerando sólo el consumo de alcohol, los sentimientos no se diferenciaron mucho, a pesar de ser mayor el número de personas. En cuanto a la calidad de la relación entre los familiares y los dependientes, de 47 (67\%) individuos, $22(46,8 \%)$ la consideran buena, $15(31,9 \%)$ regular, 6 $(12,7 \%)$ mala y $4(8,5 \%)$ pésima ${ }^{(14)}$.
La literatura apunta que, cuando los factores de riesgo a que los estudiantes están expuestos son identificados previamente durante el proceso educativo, la intervención precoz puede reducir el riesgo de futuras consecuencias de habilidades que, frecuentemente, sólo se manifestarán en el desempeño de la profesión(15). Los estudiantes de medicina que tienden a presentar, en el futuro, problemas en su carrera profesional, como consecuencia del abuso de alcohol, muchas veces inician o consumen durante la formación académica o desde antes de ingresar a la universidad(15), una vez que la prevalencia del uso es alta en esa población de estudiantes.

En México la literatura respecto al uso de alcohol en estudiantes de medicina es escasa. Sin embargo, la prevalencia del consumo de alcohol entre los mexicanos es alta y o más agravante; el patrón de consumo del alcohol es de baja frecuencia pero en grandes cantidades y con marcada tendencia a la embriaguez, caracterizando un grave problema de Salud Pública(16), datos que justifican el desarrollo del presente estudio.

El objetivo del presente trabajo es identificar el patrón del consumo de alcohol y las percepciones sobre ese consumo por personas cercanas, en una muestra de estudiantes de medicina mexicanos.

\section{Métodos}

Se trata de un estudio de tipo transversal de abordaje cuantitativo. Fue realizado en Facultad de Medicina de la Universidad Veracruzana, campus Minatitlán, Veracruz, México. Es parte del Programa de Prevención de Consumo de Drogas en Estudiantes Universitarios de esa Universidad, la cuenta con 312 estudiantes de medicina matriculados en el año de 2010. La muestra estuvo compuesta por 263 estudiantes de medicina (84.4\%).

La encuesta se realizó durante el periodo escolar de febrero a julio de 2010. Para la recolección de datos fue utilizado un cuestionario estructurado, con respuestas cerradas con información sociodemográfica, el cuestionario de estudiantes de $2006^{(17)}$ y el AUDIT ${ }^{(18)}$.

Esta prueba contiene 10 preguntas que identifican el uso de alcohol en los últimos 12 meses. Las preguntas de 4 a 6 examinan síntomas de la dependencia del alcohol, mientras que las preguntas 7 a 10 exploran las consecuencias negativas asociadas a su consumo. Cada pregunta del AUDIT tiene de tres a cinco posibles respuestas que suman al final un máximo posible de 40 puntos. Puntuación entre 0 a 7 es indicador de abstemios 
o bajo riesgo, de 8 a 12 uso de riesgo o abuso y de 13 a 40 indica probable dependencia para los hombres. Sin embargo esa clasificación puede ser realizada con dos puntuaciones siendo AUDIT <7 y AUDIT > de 8, que representa el uso problemático del consumo de

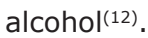

Esta Prueba presenta validez transcultural, que ha sido probada en población mexicana con una sensibilidad de $90 \%$ y especificidad del $94 \%$. Diferentes estudios la han validado en poblaciones de adolescentes, los cuales informan que es el instrumento que mejor identifica el uso y problemas o trastornos relacionados con el alcohol en adolescentes y jóvenes ${ }^{(17-20)}$

El Cuestionario de Estudiante 2006 está basado en la Encuesta Nacional de Adicciones es autoaplicable, está precodificado y contiene 158 preguntas cerradas así como indicadores que han sido elaborados por varios países, entre ellos México, conjuntamente con la Organización Mundial de la Salud. Este instrumento ha sido utilizado en diferentes encuestas con esta población, en las cuales se ha probado su validez y confiabilidad(17). Divididos en factores de riesgo para drogas y consumo de droga, sin embargo para fines de este estudio sólo se tomaron en cuenta 14 preguntas, las que se relacionan con el alcohol.

Durante la recolección de datos los estudiantes fueron orientados sobre los objetivos de la investigación respondiéndose a todo tipo de inquietudes, garantizando así el anonimato de los cuestionarios. El proyecto fue aprobado por el Comité de Ética e Investigación de la Facultad de Medicina, de la Universidad Veracruzana, campus Minatitlán, Veracruz. Para el análisis, fue diseñada una base de datos en el EPI-Info versión 6 . Empleándose frecuencia, porcentual y Chi-Cuadrado(2).

\section{Resultados}

De 312 estudiantes inscritos en la Facultad de Medicina participaron 263 (84,4\%), distribuidos por semestres: segundo $72(27,4 \%)$, cuarto $62(23,6 \%)$, sexto $38(14,4 \%)$, octavo $50(19,0 \%)$ y décimo 41 $(15.6 \%)$.

Las características sociodemográficas fueron: los estudiantes eran jóvenes con edad promedio de 20,6 (DE $\pm 1,8$ años), variando entre los 17 y 25 años, la mayoría 149 (56,7\%) mujeres y 114 (43,3\%) hombres. En relación al estado civil, 260 (98,9\%) eran solteros, declararon que pertenecen a la religión Católica $(57,4 \%)$, seguida de la Pentecostés y la Adventistas con 9,5\%. En relación con el estado civil de sus padres 199 (75.7\%) eran casados, $23(8,7 \%)$ divorciado y 20 (7,6\%) unión libre. El ingreso económico mensual de los estudiantes osciló entre 1.000 y 7.000 pesos, como presenta la Tabla 1.

Tabla 1 - Características sociodemográficas y consumo de alcohol (AUDIT), de los estudiantes de Medicina de la Universidad Veracruzana, campus Minatitlán, Veracruz, Mexico, $2010(n=263)$

\begin{tabular}{|c|c|c|c|c|c|}
\hline \multicolumn{2}{|c|}{ Variables } & \multicolumn{2}{|c|}{$\begin{array}{c}\text { AUDIT>8 } \\
\mathrm{n}=116(44,1 \%)\end{array}$} & \multicolumn{2}{|c|}{$\begin{array}{c}\text { AUDIT<7 } \\
\mathrm{n}=147(55,9 \%)\end{array}$} \\
\hline & & $f$ & $\%$ & f & $\%$ \\
\hline \multirow[t]{3}{*}{ Edad* $^{*}$} & $17-19$ * & 29 & 25,0 & 54 & 36,7 \\
\hline & $20-22$ * & 60 & 51,7 & 70 & 47,6 \\
\hline & $23-25$ * & 27 & 23,2 & 23 & 15,6 \\
\hline \multirow[t]{2}{*}{ Sexo* } & Masculino & 60 & 51,7 & 54 & 36,7 \\
\hline & Femenino & 56 & 48,3 & 93 & 63,2 \\
\hline \multirow[t]{2}{*}{ Estado civil } & Soltero & 114 & 98,2 & 146 & 99,3 \\
\hline & Unión libre & 02 & 01,8 & 01 & 00,7 \\
\hline \multirow[t]{5}{*}{ Religión } & Ninguna & 11 & 09,4 & 14 & 9,3 \\
\hline & Católica & 56 & 48,2 & 94 & 63,9 \\
\hline & Adventista & 14 & 12,1 & 11 & 07,4 \\
\hline & Pentecostés & 13 & 11,2 & 11 & 07,4 \\
\hline & Otras & 22 & 18,9 & 17 & 11,5 \\
\hline \multirow[t]{2}{*}{ Estado Civil de los padres } & Con pareja & 98 & 84,4 & 121 & 82,3 \\
\hline & Sin pareja & 18 & 15,6 & 26 & 16,7 \\
\hline \multirow[t]{3}{*}{ Ingreso personal } & $0-\$ 3.500$ & 76 & 65,5 & 105 & 71,4 \\
\hline & $\$ 3.600-15.000$ & 39 & 33,6 & 04 & 02,7 \\
\hline & $\geq \$ 15.100$ & 01 & 00,9 & 01 & 00,6 \\
\hline
\end{tabular}

*Valores $p \leq 0,05$ 
La edad de inicio del consumo de alcohol entre los estudiantes tuvo un promedio de 12,5 años (DE \pm 8.7 ) variando de 12 a 20 años.

Se encontró que la prevalencia del consumo de alcohol entre los estudiantes fue (189) $71,9 \%$ en la vida, $167(62.3 \%)$ en el último año y 142 (54\%) en el último mes.

En relación al patrón de consumo de alcohol de los estudiantes en los últimos 12 meses, la frecuencia de una o más veces al día, fue de $38.6 \%$ (44) para los hombres y $43.6 \%$ (65) para las mujeres, en cantidades de una a dos bebidas alcohólicas en un día, 33.3\% (38) hombres y $51.6 \%$ (77) mujeres. La frecuencia de consumo de 6 o más dosis de bebidas alcohólicas en una sola ocasión: menos de una vez por mes fue de $37.7 \%$ (43) para los hombres y mensual fue de $24,1 \%$ (36) para las mujeres, según se observa en la Tabla 2.

Tabla 2 - Patrón del consumo de alcohol (AUDIT) en los estudiantes de medicina de la Universidad Veracruzana campus Minatitlán, Veracruz, Mexico, 2010. $(n=263)$

\begin{tabular}{|c|c|c|c|c|c|}
\hline \multicolumn{2}{|c|}{ Variables } & \multicolumn{2}{|c|}{$\begin{array}{c}\text { Hombres } \\
114(43,3 \%)\end{array}$} & \multicolumn{2}{|c|}{$\begin{array}{c}\text { Mujeres } \\
149(56,7 \%)\end{array}$} \\
\hline & & f & $\%$ & $f$ & $\%$ \\
\hline \multirow{5}{*}{$\begin{array}{l}\text { 1. Frecuencia de consumo de } \\
\text { bebidas alcohólicas* }\end{array}$} & Nunca & 15 & 13,1 & 40 & 26,8 \\
\hline & 1 o más veces al día & 44 & 38,6 & 65 & 43,6 \\
\hline & $2-4$ veces al mes & 42 & 36,8 & 34 & 22,8 \\
\hline & $2-3$ veces a la semana & 08 & 07,0 & 08 & 05,3 \\
\hline & 4 o más veces a la semana & 05 & 04,3 & 02 & 01,3 \\
\hline \multirow{5}{*}{$\begin{array}{l}\text { 2. Cantidad de consumo de } \\
\text { bebidas alcohólicas en un día } \\
\text { normal.* }\end{array}$} & $1-2$ & 38 & 33,3 & 77 & 51,6 \\
\hline & $3-4$ & 25 & 21,9 & 22 & 14,7 \\
\hline & $5-6$ & 26 & 22,8 & 27 & 18,2 \\
\hline & $7-9$ & 17 & 14,9 & 19 & 12,7 \\
\hline & 10 o más & 08 & 07 & 04 & 02,6 \\
\hline \multirow{5}{*}{$\begin{array}{l}\text { 3. Frecuencia en que consume } 6 \\
\text { o más bebidas alcohólicas en una } \\
\text { sola ocasión.* }\end{array}$} & Nunca & 29 & 25,4 & 74 & 49,6 \\
\hline & Menos de 1 por mes & 43 & 37,7 & 31 & 20,8 \\
\hline & Mensual & 30 & 26,3 & 36 & 24,1 \\
\hline & Semanal & 10 & 8,8 & 07 & 4,6 \\
\hline & Diario o casi a diario & 02 & 1,7 & 01 & 0,6 \\
\hline
\end{tabular}

*Valores p. $\leq 0,05$

Los estudiantes referían que consumían una copa completa de bebidas alcohólicas del tipo: vino 121 (46\%), cooler y viña real 115 (44\%), cerveza 127 (48.3\%), destilados $136(51.7 \%)$, bebidas preparadas 103 (39.2\%), pulque 52 (19.8\%) y aguardiente 53 (20.2\%).

La frecuencia de consumo en el patrón de intoxicación alcohólica (o sea 6 copas o más de cualquier bebida alcohólica en una sola ocasión) se encontró: 47 (17,9\%), que no habían bebido el último año, 33 (12,5\%), por lo menos 1 vez en el último año, $20(7,6 \%)$ una vez en el último mes, $87(33,1 \%)$, de dos a tres veces en el último mes, $10(83,8 \%)$ una o más veces en la última semana, y $66(25,0 \%)$ que nunca habían bebido alcohol.

En cuanto a la frecuencia de haberse emborrachado, $67(25,5 \%)$ de los estudiantes nunca en el último año,
$28(10,6 \%)$ por lo menos 1 vez en el último año, 90 $(34,2 \%)$ una vez en el último mes, $2(0,8 \%)$ de dos a tres veces en el último mes, $6(2,3 \%)$ una o más veces en la última semana.

El estudiante percibe que el consumo de alcohol es evaluado por el Padre, la Madre, y los Maestros y todos lo ven mal, teniendo significancia estadística en los maestros $p=\leq 0,05$. Al evaluar la percepción del consumo de bebidas alcohólicas por los familiares, maestros y amigos, $85 \%$ lo ve mal, la madre o quien la sustituya, $83,7 \%$ el padre o quien lo sustituya, $84,0 \%$ sus maestros y $38,8 \%$ sus mejores amigos. La percepción del peligro respecto al consumo del alcohol fue referida por el $72.2 \%$ como muy peligroso como se observa en la Tabla 3. 
Tabla 3 - Percepciones y niveles de consumo de alcohol (AUDIT) en los estudiantes de medicina de la Universidad Veracruzana campus Minatitlán, Veracruz, Mexico, 2010. $(n=263)$

\begin{tabular}{|c|c|c|c|c|c|c|}
\hline & \multicolumn{2}{|c|}{ Variables } & \multicolumn{2}{|c|}{$\begin{array}{c}\text { AUDIT>8 } \\
n=116(44,1 \%)\end{array}$} & \multicolumn{2}{|c|}{$\begin{array}{c}\text { AUDIT<7 } \\
\mathrm{n}=147(55,9 \%)\end{array}$} \\
\hline & & & f & $\%$ & $f$ & $\%$ \\
\hline \multirow{9}{*}{$\begin{array}{l}\text { Percepción del } \\
\text { consumo de alcohol }\end{array}$} & \multirow[t]{2}{*}{ Madre } & Ni bien ni mal & 14 & 19,1 & 24 & 16,3 \\
\hline & & Mal & 102 & 87,9 & 123 & 83,7 \\
\hline & \multirow[t]{2}{*}{ Padre } & Ni bien ni mal & 20 & 17,2 & 23 & 15,6 \\
\hline & & Mal & 96 & 82,8 & 124 & 84,3 \\
\hline & \multirow[t]{2}{*}{ Maestro $(a)^{*}$} & Ni bien ni mal & 09 & 07,7 & 33 & 22,5 \\
\hline & & Mal & 107 & 92,2 & 114 & 77,5 \\
\hline & \multirow[t]{3}{*}{ Amigo (a) } & Lo vería bien & 02 & 01,8 & 09 & 06,1 \\
\hline & & Ni bien ni mal & 72 & 63,0 & 75 & 51,0 \\
\hline & & Mal & 39 & 33,6 & 63 & 42,8 \\
\hline \multirow{3}{*}{$\begin{array}{l}\text { Percepción del peligro } \\
\text { de consumir alcohol }\end{array}$} & & No es peligroso & 06 & 05,1 & 03 & 2,04 \\
\hline & & Es peligroso & 24 & 20,6 & 40 & 27,2 \\
\hline & & Es muy peligroso & 86 & 74,1 & 104 & 70,7 \\
\hline
\end{tabular}

*Valores $\mathrm{p} \leq 0,05$

La presencia de problemas familiares debido al consumo del alcohol ocurrieron en $21,3 \%$, siendo el padre $20(7,6 \%)$, la madre $4(1,5 \%)$, hermano (a) 6 $(2,3 \%)$ y otro pariente cercano $30(11,4 \%)$

Con respecto a peleas en la familia sólo $21(8,0 \%)$ respondieron afirmativamente. Cuando se les pregunta si esta pelea estuvo asociada al consumo de alcohol, 18 $(6,8 \%)$ respondieron que si.

En relación a los niveles de riesgo (AUDIT), 142 (54\%) estudiantes consumían en nivel de riesgo o eran abstinentes, como se observa en el Tabla 4.

Tabla 4 - Nivel de consumo de alcohol (AUDIT), según los estudiantes de medicina, Universidad Veracruzana campus Minatitlán, Veracruz, Mexico, 2010. $(n=263)$

\begin{tabular}{lcc}
\hline \multicolumn{1}{c}{ Niveles de riesgo } & f & $\%$ \\
\hline Bajo riesgo o abstinentes & 142 & 54,0 \\
De riesgo o abuso & 59 & 22,4 \\
Riesgo de probable dependencia de alcohol & 62 & 23,6 \\
\hline
\end{tabular}

\section{Discusión}

La muestra de este estudio fue compuesta por 263 estudiantes de medicina, en su mayoría por mujeres, jóvenes, solteros y de religión católica, con un ingreso personal entre 0 a 3.500 pesos mensuales y con padres que conviven en pareja, datos similares a los de otros estudios realizados con estudiantes de medicina(4-5,12).

La prevalencia del consumo de alcohol está muy presente en la vida de estos jóvenes, futuros profesionistas, $71,9 \%$ usó en la vida, $62,3 \%$ en el último año y $54 \%$ en el último mes. Sin embargo, esta es una realidad presentada en diversos estudios internacionales ${ }^{(6-}$ ${ }^{11)}$ y también en diferentes países de América Latina ${ }^{(6-10)}$ que utilizaron el mismo instrumento.

En relación a la edad de inicio de consumo se identificó que el uso experimental se presentó de forma bastante precoz (12,5 años). Esa realidad no ha sido diferente en la población mexicana ${ }^{(4,21)}$ y también en estudios que señalan que el uso se ha presentado en edades cada vez más tempranas(22-23), justificado por el papel socializador que esa sustancia proporciona(21).

En relación al patrón de consumo, se observa que el consumo en ambos sexos ha sido de baja frecuencia y cantidades. Este resultado llama la atención, ya que la mayoría son mujeres que sobresalen en el consumo. Sin embargo, el consumo de modo a emborracharse (más de 6 o más dosis en una sola ocasión) fue más frecuente entre los hombres $(37,7 \%)$.

Estos datos corroboran con los de la literatura(24), una vez que los mexicanos consumen en bajas frecuencia pero en grandes cantidades y con marcada tendencia a la embriaguez ${ }^{(4)}$, Dato que se torna preocupante frente a las posibles consecuencias (sociales y de salud) que pudieran resultar.

La literatura evidencia que ese patrón de consumo es característico del sexo masculino(25) y bastante discutido en la literatura, por haber sido reconocido como una característica cultural distinta al patrón de beber de cada país, pudiendo tener significados diferentes. El consumo en el patrón de intoxicación se ha tornado un problema de interés social, político y de salud en diversos países, 
debido a sus graves consecuencias para el individuo y las comunidades con altos costos sociales(23).

En el tipo de bebida alcohólica, las más consumidas fueron las destiladas y la cerveza. El tipo de bebida se caracteriza como un aspecto importante del patrón de consumo y por otro lado, la bebida escogida puede influenciar en los riesgos y acabar resultando en problemas.

En relación a los niveles de riesgo referente al consumo de alcohol, fue identificado en el presente estudio que $46 \%$ de los estudiantes consumían en niveles problemáticos y que es posible realizar intervenciones. Debido a las diversas culturas de consumo de alcohol en estudiantes de medicina, de diferentes países, inclusive utilizando el mismo instrumento y puntuaciones, se torna compleja una comparación, pero los resultados se asemejan ${ }^{(4)}$, en algunos países latinos son bastantes superiores $^{(11,21-22)} \mathrm{o}$ inferiores ${ }^{(24)}$.

En cuanto la percepción de riesgo a respecto del consumo de bebidas alcohólicas, la mayoría de los estudiantes de medicina percibe que sus padres evalúan el uso como muy peligroso. También perciben de forma negativa la evaluación por los familiares y amigos cuando estos perciben el consumo de bebidas alcohólicas, con estadística significativa. De lo que se puede deducir que el consumo, en casa, por familiares o amigos ha sido nuevamente un comportamiento social más permitido. Sin embargo, cuando se trata de los maestros, que muchas veces tienen que mostrarse como un modelo de referencia, el consumo de alcohol no siempre es bien visto por ellos ${ }^{(12-25)}$.

En los familiares que tienen problemas con el uso de alcohol, los estudiantes refirieron que el padre $(7,6 \%)$ fue la persona que más presentó problemas. El uso de alcohol puede interferir en las relaciones afectivas y estructurales de la familia tornándose un factor de riesgo que influye en el consumo de bebidas alcohólicas entre los jóvenes. Algunas evidencias demuestran que la familia es el circulo social en que ocurren todas las relaciones afectivas y el principal contexto de aprendizaje de un individuo, de modo que una persona depende en gran medida del buen funcionamiento de la situación familiar(24-25). Sin embargo, el presente estudio mostró que una minoría ( $8 \%$ ) de los estudiantes relató que hay pleitos familiares como consecuencia del consumo del alcohol, y que parece ser un indicador de factores de protección frente al uso de alcohol para esta muestra.

En razón del diseño metodológico existen limitaciones en el presente estudio. Esta es la primera vez que se evalúa un grupo de estudiantes de medicina mexicanos. Otros estudios de seguimiento son necesarios para conocer más, específicamente, el consumo de alcohol en este grupo de población y con instrumentos que permitan realizar un diagnóstico más apropiado del problema del uso de alcohol especialmente entre las mujeres.

\section{Conclusión}

Este estudio buscó conocer el patrón de consumo de alcohol en una muestra de estudiantes de medicina mexicanos. Se trata de un tema preocupante que debe ser priorizado con acciones de prevención frente al uso de alcohol, una vez que se caracteriza por ser una población joven que formará futuros profesionales de la salud.

Además, se identificó un consumo problemático de bebidas alcohólicas en casi la mitad de los estudiantes y en el patrón de intoxicación entre los hombres. El consumo de alcohol fue evaluado como algo peligroso. Los estudiantes tienen la percepción que los maestros ven muy mal que ellos consuman bebidas.

El consumo de alcohol está presente en los problemas entre los familiares, principalmente por el padre; un pequeño porcentaje de alumnos presenciaron peleas en la familia que resultaron como consecuencia del uso del alcohol.

Los resultados podrán contribuir para la planificación de programas y acciones preventivas en relación al consumo de bebidas alcohólicas en el ámbito universitario.

\section{Agradecimientos}

Agradecemos a la Comisión Interamericana para el Control del Abuso de Drogas/CICAD de la Secretaria de Seguridad Multidimensional/SSM de la Organización de los Estados Americanos/OEA, la Secretaria Nacional de Políticas sobre Drogas/SENAD do Gabinete de Seguridad Institucional/Brasil, la Escuela de Enfermería de Ribeirao Preto de la Universidad de Sao Paulo y Centro Colaborador de la Organización Mundial de la Salud para el Desarrollo de la Investigación en Enfermería, la población representada en los estudios de investigación, bien como a las autoridades de las universidades representadas por los participantes del Programa En-Line de Especialización en Investigación sobre el Fenómeno de las Drogas, periodos 2006, 2007, 2008 y 2009. 


\section{Referencias}

1. Bolet-Astovizay $\mathrm{M}$, Socarrás-Suárez MM. El alcoholismo, consecuencias y Prevención. Rev Cubana Invest Biomed. 2003;22(1):25-31.

2. Secretaria de Salud (MX). Encuesta Nacional de Adicciones (ENA-2008) México, D.F: Dirección General de Epidemiología/Instituto Mexicano de Psiquiatría; 2008.

3. Medina MM, Villatoro J, Cravioto P, Fleiz C, Galvan F. Uso y abuso de alcohol en México: Resultado de la Encuesta Nacional Contra las Adicciones. En: Consejo Nacional Contra las adicciones, editor. Observatorio Mexicano en tabaco, alcohol y otras drogas. México: Consejo Nacional Contra las Adicciones; 2003. p. 49-61.

4. Díaz MA, Díaz MLR, Hernández A, Narro RJ, Hernández $H$, Solís TC. Prevalencia del consumo riesgoso y dañino de alcohol y factores de riesgo en estudiantes universitarios de primer ingreso. Rev Salud Mental. 2008;31:271-82. 5. Medina-Mora ME, Natera G, Borges G, Cravioto $P$, Fleiz C, Tapia-Conyer R. Del siglo XX al tercer milenio. Las Adicciones y la Salud Pública: Drogas, Alcohol y Sociedad. Rev Salud Mental. 2001;24(4):3-19.

6. Pinton FA, Boskovitz EP, Cabrera EMS. Uso de drogas entre os estudantes de medicina da Faculdade de Medicina de São José do Rio Preto, SP, no ano de 2002. Arq Ciênc Saúde. 2005,12(2):91-6.

7. Soprani PJD, Santos de Souza R, Buaiz V, Miguel SM. Uso de substâncias psicoativas entre universitários de medicina da Universidade Federal do Espírito Santo. Rev Bras Psiquiatria. 2008;57(3):188-95.

8. Tockus D, Gonçalves PS. Detecção do uso de drogas de abuso por estudantes de medicina de uma universidade privada. J Bras Psiquiatria. 2008;57(3):184-7.

9. Montoya VEM, Cunningham J, Brands B, Carol Strike C, Miotto WMG. Consumo percibido y uso de drogas lícitas e ilícitas en estudiantes universitarios en la Ciudad de Medellín, Colombia Rev. Latino-Am. Enfermagem. 2009;17(no esp):996-92.

10. Vergara KMA. Consumo patológico de alcohol entre los estudiantes de la Universidad de Cartagena 2008. Rev Salud Pública. 2009;11(6):878-86.

11. Boland M, Fitzpatrick P, Scallan E, Daly L, Herity B, Horgan J, Bourke G. Trends in medical student use of tobacco, alcohol and drugs in an Irish university, 19732002. Drug Alcohol Depend. 2006;85(2):123-8.

12. Peuker AC, Fogaça J, Bizarro L. Expectativas e beber problemático entre universitários. Psicologia. 2006;22(2):193-200.

13. Rojas-Guit E, Fleiz- Bautista C, Medina-Mora IME, Moron ML, Domenech-Rodriguez M. Consumo de alcohol y drogas en estudiantes de Pachuca, Hidalgo. Rev Salud Pública México. 1999;(4):297-308.
14. Martins $M$; Santos MA, Pillon SC. Percepciones de familias con bajos ingresos sobre el consumo de drogas por un miembro de su familia. Rev. Latino-Am. Enfermagem. 2008;16(2):293-8.

15. Sáez MN, Medina V, Romaguera F, Ruza EC, Rodríguez $A$. Fatores de risco e propostas para a redução da demanda de drogas em estudantes de medicina de uma universidade venezuelana. SMAD, Rev. Eletrônica Saúde Mental Álcool Drog. (Ed. Port) [internet]. 2009, 5(2) [acesso 20 set 2010]: 1-16. Disponível em: http:// pepsic.bvsalud.org/scielo.php?k;5(2):1-16.

16. Herrera-Vazquez $M$, Wagner FA, Velasco-Mondragon E, Borges G, Lascano-Ponce E. Inicio en el consumo de alcohol y tabaco y transición a otras drogas en adolescentes de Morelos, México. Salud Publica México. 2004;46(2):132-40.

17. Medina-Mora ME, Castro S, Campillo-Serrano C, Gómez-Mont FA. Validity and reliability of a high school drug use questionnaire among Mexican students. Bull Narc. 1981;33(4):67-76.

18. Saunders JB, Aasland OG, Babor TF, de la Fuente JR, Grant M. Development of the Alcohol Use Disorders Screening Test (AUDIT). WHO collaborative project on early detection of persons with harmful alcohol consumption. Addiction. 1993;88:791-804.

19. Alvarado ME, Garmendia ML, Acuña G, Santis R, Arteaga O. Validez y Confiabilidad de la versión Chilena del Alcohol Use Disorders Identification Test (AUDIT). Rev Med Chile. 2009;137:1463-9.

20. O'Hare T, Sherrer MV. Validating the Alcohol Use Disorder Identification Test With College First-Offenders. J Substance Abuse Treat. 1999;17(1-2):113-9.

21. Mora-Rios J, Natera G. Expectativas de consumo de alcohol y problemas asociados en estudiantes universitarios de la ciudad de México. Salud Pública de México. 2001;43:89-96.

22. Londoño PC, García HW, Valencia LSC, Vinaccia AS. Expectativas frente al consumo de alcohol en jóvenes universitarios colombianos. Anales Psicol. 2005;21(2):259-67.

23. Arrieta VKM. Consumo patológicos de alcohol entre estudiantes de la Universidad de Cartagena 2008. Rev Salud Pública. 2009;11(6):878-86.

24. Ardila MF, Herrán OF. Expectativas en el consumo de alcohol en Bucaramanga, Colombia. Rev Méd Chile. 2008;136:73-82.

25. Zamboanga BL. Alcohol expectancies and drinking behaviors in Mexican American college students. Addictive Beh. 2005;30:673-84.

Recibido: 21.2.2011

Aceptado: 13.4.2011 